\title{
Post-traumatic stress disorder and attention- deficit hyperactivity disorder in Palestinian children affected by the war on Gaza
}

\author{
Abdel Aziz Thabet, ${ }^{1}$ Abu Tawahina, ${ }^{2}$ Eyad El Sarraj, ${ }^{3}$ David Henely, ${ }^{4}$ \\ Henrick Pelleick ${ }^{5}$ and Panos Vostanis ${ }^{6}$
}

'Senior Researcher, Gaza Community Mental Health Programme, Gaza, Palestine; ${ }^{2}$ Clinical Psychologist, Gaza Community Mental Health Programme; ${ }^{3}$ Consultant Psychiatrist, Gaza Community Mental Health Programme; ${ }^{4}$ Associate Professor, Uppsala University, Sweden; ${ }^{5}$ Associate Professor, Uppsala University, Sweden; ${ }^{6}$ Professor of Child Psychiatry, Greenwood Institute of Child Health, University of Leicester, UK, email pv11@le.ac.uk

\begin{abstract}
he Gaza Strip has been badly affected by war and conflict over the past four decades. Palestinians are exposed to repeated incursions of the border areas, with many resulting casualties. At the end of December 2008, more than 230 Palestinians were killed and at least 770 were injured, including 100 in critical condition, after an Israeli airstrike. This was followed by a military operation that continued for 23 days, in which 1420 Palestinians, including 446 children, were killed. At least 4000 houses were totally destroyed and 16000 partially damaged (Palestinian Red Crescent, 2008).

Previous studies have reported on the impact of trauma on child and adult mental health following this conflict (Thabet et al, 2008). Indeed, there is a growing literature on the prevalence of emotional disorders, predominantly posttraumatic stress disorder (PTSD) and depression, in similar circumstances. Some studies have focused on the different pathways and mechanisms between these disorders, while others have examined their comorbidity. Less research has been done on the comorbidity between PTSD and externalising conditions such as attention-deficit hyperactivity disorder (ADHD). This would be particularly important for diagnostic and treatment purposes, because of the potential similarities between symptoms such as hyperarousal and hypervigilance (Ford et al, 2000). For example, an early study of maltreated children found that 35\% met criteria for PTSD, of whom 37\% were also diagnosed with ADHD (Famularo et al, 1996). The rationale of the present study was to examine this interesting type of comorbidity in children at high risk of developing PTSD because of their exposure to war trauma.
\end{abstract}

\section{Method}

The sample was randomly selected from the population census in five areas of the Gaza Strip. Each area was divided into blocks; from each block one street was chosen; and from each street every tenth family home was approached. All selected families provided informed consent. The resulting sample consisted of 410 Palestinian children, aged 8-18 years (mean 12.9, s.d. 3.9): 224 boys (54.6\%) and 186 girls. The fieldwork was conducted between May and June 2009 by five mental health professionals trained in research.

The following measures were completed:
O War on Gaza Traumatic Events Checklist for Children (Thabet et al, 2008). The earlier version was adapted for the nature of traumatic events occurring during the recent conflict. It consisted of 25 items, rated 'no/yes' (0/1), and a total score was calculated (Cronbach's $\alpha=0.80$, split half $=0.68$ ).

O UCLA PTSD Index for DSM-IV for Children (Pynoos et al, 1998). This comprises 22 self-report items rated $0-4$. A total score was calculated and a categorical prevalence estimated. The reliability of the Arabic version used in this study was ascertained (Cronbach's $\alpha=0.83$, split half $=0.81$ ).

O Structured Clinical Interview for DSM-IV Diagnosis of ADHD - Parents' form - Arabic version (Thabet et al, 2006). This consists of 18 items, with children scoring positive on six or more 'inattentive' items considered inattentive and those positive on six or more 'hyperactivity-impulsivity' items considered hyperactiveimpulsive. The 'combined' category (inattentive-impulsive) is based on summing both inattentive and hyperactiveimpulsive scores. The Arabic version was used, following translation and back-translation by a panel of five experts (Cronbach's $\alpha=0.89$, split half $=0.760$ ).

\section{Results}

Families lived in cities (43.5\%), villages (12.5\%) and refugee camps (44.0\%). Their size was large, as $13.6 \%$ had fewer than four children, $61.4 \%$ had five to seven children and $25.0 \%$ eight or more children. The majority $(60.9 \%)$ had a monthly income of less than US\$350, 30.4\% earned US\$351-700 and only $8.7 \%$ earned more than US\$700.

Palestinian children reported a range of traumatic experiences: $95 \%$ had heard sonic sounds of jetfighters, $92 \%$ had heard shelling by artillery, 92\% had seen mutilated bodies on television, $80 \%$ had been deprived of water or electricity during the war and $51 \%$ had left their home for a safer place. The mean number of reported traumatic events was 8.5 (s.d. 3.9), without significant gender differences $(t=1.18, P=0.23)$.

The total number of traumatic events (as recorded on the Traumatic Events Checklist) was significantly associated with scores on the UCLA PTSD Index (Pearson correlation $r=0.29$, $P=0.001)$. There was no significant difference between 
boys and girls in PTSD scores $(t=0.53, P=0.56)$. Overall, $39.3 \%$ of children reported reactions that fulfilled the DSM-IV criteria for partial PTSD, while a further $9.8 \%$ met the criteria for full PTSD. According to parents' reports on the Structured Clinical Interview for DSM-IV Diagnosis of ADHD, $31.3 \%$ of children reported clinically significant attentiondeficit symptoms, 36.3\% reported impulsivity-hyperactivity symptoms and $29.0 \%$ reported a combination of the two (inattentive-impulsive). Twenty-one children (5.1\%) had significant comorbid PTSD and attention-deficit symptoms, 18 (4.4\%) had comorbid PTSD and impulsivity-hyperactivity symptoms and 18 children (4.4\%) had comorbid PTSD and inattentive-impulsive symptoms.

\section{Discussion}

This study was conducted 6 months after the participants had been exposed to a wide range of traumatic events during the war on Gaza. Most of the children still remembered traumatic events and over half had had to leave their home for a place of safety. This is consistent with our previous studies (Thabet et al, 2008).

In the present study, $39.3 \%$ of children reported partial PTSD and $9.8 \%$ reported full PTSD, which is also consistent with earlier findings. Pat-Horenczyk et al (2009) assessed the effects of ongoing violence on the mental health of Palestinian and Israeli youths. They found that $37.2 \%$ of Palestinian students in the West Bank reported symptoms meeting the criteria for full PTSD and an additional $12.1 \%$ reported symptoms meeting the criteria for partial PTSD. Abdeen et al (2008), in a study of Palestinian students from both the West Bank and Gaza, similarly found that $36 \%$ of West Bank and $35 \%$ of Gaza participants reported symptoms meeting criteria for full PTSD according to DSM-IV-TR, while 12\% and 11\% respectively reported symptoms meeting criteria for partial PTSD.

The focus of the present study was, however, the overlap or comorbidity between post-traumatic stress and attention-deficit hyperactivity, which ranged between $4.4 \%$ and $5.1 \%$ for the ADHD symptom categories recorded on the Structured Clinical Interview for DSM-IV Diagnosis of ADHD. The overlap of ADHD and PTSD often involves the following symptoms: irritability, excessive motor activity, concentration difficulties, impulsive behaviour and exaggerated startle responses (American Psychiatric Association, 1994). Both conditions may be characterised by difficulty in concentrating, restlessness or irritability and impulsivity. Some authors (e.g. Famularo et al, 1996) have argued that because several symptoms in the diagnosis of ADHD also arise in the context of the maltreated child syndrome (or attachment disorders), they may indicate PTSD and anxiety rather than developmental disorder. Alternatively, physical overactivity in children with PTSD could be a result of increased muscular tension because of anxiety. According to DSM-IV-TR (American Psychiatric Association, 2000), the symptoms of ADHD and PTSD in growing children have certain similarities. In both ADHD and PTSD there is difficulty in sustaining attention, an incapacity to listen, failure to complete duties, difficulty in organisation, avoidance of mental tasks, memory dysfunction, irritability and restlessness.

Children repeatedly exposed to traumatic events seem to have a high risk of displaying both sets of symptoms and diagnoses. The finding of PTSD-related and ADHD-related symptoms appearing together in the same child might be looked on as comorbidity. Nevertheless, true comorbidity might not apply because the symptoms manifested in the two disorders are so similar. Children may have PTSD-related symptoms and, because of their anxious state, show hyperactivity, attention problems and impulsivity. This could be conceptualised as ADHD by the clinician.

The diagnostic implications would benefit from further research with children exposed to different types of trauma, especially longitudinal studies that might disentangle the phenomenology and outcome of the two disorders (including their comorbid presentation). Whatever the underlying issues and mechanisms, the findings highlight the importance of psychiatric training in detecting both the effects of trauma and neurodevelopmental disorders, across different conflict situations and cultural groups. Only accurate diagnosis can formulate appropriate treatment for children with $A D H D$, as opposed to psycho-educational and other universal and targeted interventions for PTSD.

It is important to acknowledge a number of limitations in this study, such as: the use of structured interviews in the community that were originally designed to measure childhood ADHD in clinical settings; the lack of multi-informant and consistent corroboration of both PTSD and ADHD symptoms; and the lack of measurement at different points.

\section{Acknowledgements}

We are grateful to all children and families who participated in the study, and to the five mental health practitioners who completed the fieldwork.

\section{References}

Abdeen, Z., Qasrawi, R., Nabeel, S., et al (2008) Psychological reactions to Israeli occupation: findings from the national study of school-based screening in Palestine. International Journal of Behavioral Development, 32, 290-297.

American Psychiatric Association (1994) Diagnostic and Statistical Manual of Mental Disorders (4th edn) (DSM-IV). APA.

American Psychiatric Association (2000) Diagnostic and Statistical Manual of Mental Disorders (4th edn, text revision) (DSM-IV-TR). APA.

Famularo, R., Fenton, T., Kinscherff, R., et al (1996) Psychiatric comorbidity in childhood posttraumatic stress disorder. Child Abuse and Neglect, 20, 953-961.

Ford, J., Racusin, R., Ellis, C., et al (2000) Child maltreatment, other trauma exposure, and posttraumatic symptomatology among children with oppositional defiant and attention deficit hyperactivity disorders. Child Maltreatment, 5, 205-217.

Palestinian Red Crescent (2008) Humanitarian Duty Report. Palestinian Red Crescent.

Pat-Horenczyk, R., Qasrawi, R., Lesack, R., et al (2009) Posttraumatic symptoms, functional impairment and coping among adolescents on both sides of the Israeli-Palestinian conflict: a cross-cultural approach. Applied Psychology: An International Review, 58, 688-708.

Pynoos, R., Rodriquez, N., Steinberg, A., et al (1998) The UCLA Post Traumatic Stress Reaction Index for DSM-IV. UCLA Trauma Psychiatric Program.

Thabet, A. A., Abdulla, T., El Helou, M., et al (2006) Effect of trauma on children's mental health in the Gaza Strip and the West Bank. In Protection of Children During Armed Political Conflict: A Multidisciplinary Perspective (eds C. Greenbaum, P. Veerman \& N. Bacon-Shnoor), pp. 123-138. Intersentia Publishers.

Thabet, A. A., Abu Tawahina, A., El Sarraj, E., et al (2008) Exposure to war trauma and PTSD among parents and children in the Gaza Strip. European Child and Adolescent Psychiatry, 17, 191-199. 